\title{
Effective Potential for Ultracold Atoms at the Zero Crossing of a Feshbach Resonance
}

\author{
N. T. Zinner ${ }^{1,2}$ \\ ${ }^{1}$ Department of Physics, Harvard University, Cambridge, MA 02138, USA \\ ${ }^{2}$ Department of Physics and Astronomy, University of Aarhus, 8000 Aarhus, Denmark
}

Correspondence should be addressed to N. T. Zinner, zinner@phys.au.dk

Received 25 February 2012; Revised 25 June 2012; Accepted 26 June 2012

Academic Editor: Ali Hussain Reshak

Copyright (C) 2012 N. T. Zinner. This is an open access article distributed under the Creative Commons Attribution License, which permits unrestricted use, distribution, and reproduction in any medium, provided the original work is properly cited.

\begin{abstract}
We consider finite-range effects when the scattering length goes to zero near a magnetically controlled Feshbach resonance. The traditional effective-range expansion is badly behaved at this point, and we therefore introduce an effective potential that reproduces the full $T$-matrix. To lowest order the effective potential goes as momentum squared times a factor that is well defined as the scattering length goes to zero. The potential turns out to be proportional to the background scattering length squared times the background effective range for the resonance. We proceed to estimate the applicability and relative importance of this potential for Bose-Einstein condensates and for two-component Fermi gases where the attractive nature of the effective potential can lead to collapse above a critical particle number or induce instability toward pairing and superfluidity. For broad Feshbach resonances the higher order effect is completely negligible. However, for narrow resonances in tightly confined samples signatures might be experimentally accessible. This could be relevant for suboptical wavelength microstructured traps at the interface of cold atoms and solid-state surfaces.
\end{abstract}

\section{Introduction}

Cold atomic gases have enjoyed many great successes since the first realizations of Bose-Einstein condensates in the mid nineties [1]. Ensembles of ultracold atomic gases can be manipulated in magnetic or optical trap geometries and in lattice setups, effectively mimicking the structure of real materials and teaching us about their properties. In particular, extreme control can be exercised over the atomatom interactions through the use of Feshbach resonance [2]. Tuning the system into the regime of resonant two-body interactions provides a controlled way of studying strongly correlated dynamics which is believed to be crucial for material properties such as high-temperature superconductivity or giant magnetoresistance.

Recently there has been extended interest in weakly interacting Bose-Einstein condensates for use as an atomic interferometer [3] and also to probe magnetic dipolar interactions in condensates [4]. This work was based on ${ }^{39} \mathrm{~K}$ atoms where a broad Feshbach resonance exists at a magnetic field strength of $B_{0}=402.4 \mathrm{G}$ [5] which allows a large tunability of the atomic interaction in experiments [6]. Similar tunability has also been reported in a condensate of ${ }^{7} \mathrm{Li}$ [7]. The atomic interaction can be reduced by tuning the scattering length, $a$, to zero, also known as zero crossing. In a Gross-Pitaevskii mean-field picture we can thus neglect the usual nonlinear term proportional to $a$. The question is then what other interactions are relevant. As shown in [4], the magnetic dipole will contribute here.

In the Gross-Pitaevskii picture we might also ask whether higher order terms in the interaction can contribute around zero crossing. Recently it was shown that effective-range corrections can in fact influence the stability of condensates around zero crossing [8-10]. The Feshbach resonances used thus far in experiments have typically been very broad, and as a result the effective range, $r_{e}$, will be small, rendering the higher order terms negligible. However, around narrow resonances this is not necessarily the case and finite-range corrections are not necessarily negligible.

For the two-component Fermi gas, there has been increased interest in producing a cold atom analog of the celebrated Stoner model of ferromagnetism [11] which 
applies to repulsively interacting fermions. Theoretical proposals indicate that this should be possible [12-19], and an MIT experiment subsequently announced indications of the ferromagnetic transition [20]. The results caused controversy since the spin domains were not resolved [21-24]. A later experiment in the same group did not find evidence of the ferromagnetic transition [25]. However, these studies consider broad Feshbach resonances, and the situation with narrow resonances is less clear. One can imagine that finiterange corrections could play a role in driving the phase transition. In fact, a recent experiment in Innsbruck [26] has found increased lifetimes of the repulsive gas in the strongly imbalanced case, providing hope that decay into molecules can be controlled and ferromagnetism can be studied.

The systematic inclusion of finite-range effects through derivative terms in zero-range models was begun in the study of nuclear matter decades ago $[27,28]$. Later on the intricacies of the cut-off problems that arise in this respect were considered by many authors both for the relativistic and nonrelativistic case (see [29] for discussion and references). In the context of cold atoms and Feshbach resonances, we need to use a two-channel model [30] in order to take the lowest order finite-range term into account. Similar models were already introduced in [31] and denoted resonance models (see f.x. [32] for a comprehensive review of scattering models for ultracold atoms). We note that whereas resonance models treat the closed-channel molecular state as a point boson the model of [30] treats the molecule more naturally as a composite object of two atoms. In the end the parameters of the two models turn out to be similarly related to the physical parameters of Feshbach resonances (see for instance the discussion of resonance models in [32]).

In Figure 1 we show calculations of scattering length and effective range for the Feshbach resonance at $B=202.1 \mathrm{G}$ in ${ }^{40} \mathrm{~K}$ in both a coupled-channel model [33] and in the zero-range model discussed here. We see the effective range being roughly constant at resonance and then start to diverge at zero crossing. The zero-range model provides a good approximation to the full calculations and for many-body purposes it is preferable due to its simplicity.

Whereas the earlier work of [31] considered the regime close to the resonance, we will be exclusively concerned with zero crossing. To our knowledge the intricacies of this region have not been addressed in the literature in the context of Feshbach resonances. Around zero crossing the Feshbach model turns out to have a badly behaved effective-range expansion. The parameters obtained from the effective-range expansion should therefore be used with extreme caution as the series is divergent at this point. However, as we show in this paper, the finite-range corrections obtained from the full $T$-matrix at low momenta via an effective potential turn out to be the same as one would naively expect based on the effective-range expansion. After introducing the effective potential we consider its applicability and importance in the case of Bose-Einstein condensates and for two-component Fermi gases where the attractive nature of the effective interaction at zero crossing could lead to collapse above a certain critical particle number or to pairing instability and superfluidity. In general, we find that tight external

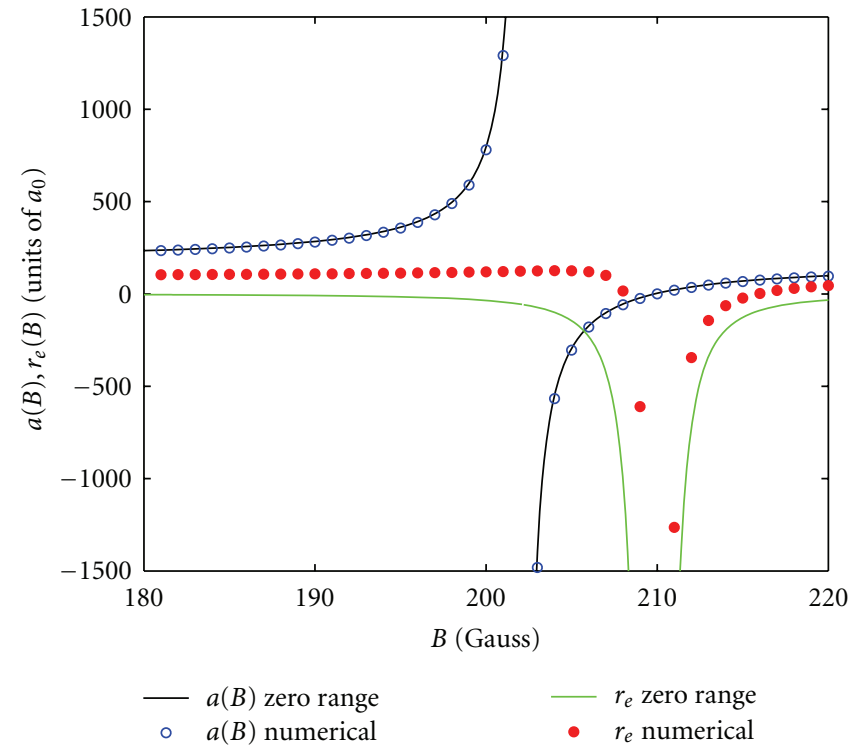

Figure 1: Scattering length and effective range for the $s$-wave scattering of fermionic ${ }^{40} \mathrm{~K}$ atoms around the Feshbach resonance at $B_{0}=202.1 \mathrm{G}$ demonstrating the divergence in a coupled-channel calculation (symbols) [33] and in a zero-range model (full lines). The difference in the zero-range and coupled-channel models is caused by the presence of a bound state close to threshold in the open channel.

confinement is a necessary condition for the higher order effects to dominate the magnetic dipole interaction and be experimentally observable.

\section{Two-Channel Model}

We consider a two-channel $s$-wave Feshbach model with zero-range interactions [30] for which the on-shell openopen channel $T$-matrix as a function of magnetic field, $B$, is

$$
T_{o o}(B)=\frac{\left(4 \pi \hbar^{2} / m\right) a_{b g}}{\left(1+\Delta \mu \Delta B /\left(\hbar^{2} q^{2} / m-\Delta \mu\left(B-B_{0}\right)\right)\right)^{-1}+i a_{b g} q},
$$

where $\Delta \mu$ is the difference between the magnetic moments in the open and closed channel, $q$ is the relative momentum of the atoms of mass $m, a_{b g}$ is the scattering length away from the resonance at magnetic field $B_{0}$, and $\Delta B$ is the width of the resonance. We can compare this to the standard vacuum expression for the $T$-matrix in terms of the phase-shift given by

$$
T_{\nu}=\frac{\left(4 \pi \hbar^{2} / m\right) a}{-q a \cot \delta(q)+i a q} .
$$

Typically, one has the low-energy expression $-q \cot \delta(q)$ $\rightarrow-1 / a$ which implies that

$$
T_{\nu} \longrightarrow \frac{\left(4 \pi \hbar^{2} / m\right) a}{1+i a q} \longrightarrow 0
$$


However, as we now discuss, for the realistic two-channel $T$-matrix for Feshbach resonances, the quantity $-q \cot \delta(q)$ is not well defined, and the conclusion that the $T$-matrix vanishes at zero crossing is only true for zero momentum, $q=0$, as we now discuss.

From (1) and (2) we obtain the relation for the phaseshift:

$$
q \cot \delta(q)=\frac{-1}{a_{b g}}\left(1+\frac{\Delta \mu \Delta B}{\left(\hbar^{2} q^{2} / m-\Delta \mu\left(B-B_{0}\right)\right)}\right)^{-1} .
$$

We now expand the right-hand side in powers of $q$ as is usually done in an effective-range expansion. This yields

$$
\begin{aligned}
q \cot \delta(q)= & \frac{-1}{a(B)} \\
& +\sum_{n=1}^{\infty} \frac{-1}{a_{b g}}\left[\frac{-a_{b g} r_{e 0}}{2}\right]^{n}\left[\frac{a_{b g}}{a(B)}-1\right]^{n+1} q^{2 n},
\end{aligned}
$$

where $a(B)=a_{b g}\left(1-\Delta B /\left(B-B_{0}\right)\right)$ is the common parametrization from single-channel models and $r_{e 0}=$ $-2 \hbar^{2} /\left(m \Delta B \Delta \mu a_{b g}\right)$ is the background value of the effective range around the resonance. From (5) we can now read off all coefficients in an effective-range expansion with their full $B$-field dependence. For instance, the effective range is given simply by $r_{e}=r_{e 0}\left[\left(a_{b g} / a\right)-1\right]^{2}$, which is divergent when $a(B) \rightarrow 0$. We also clearly see that all the other coefficients are divergent in that limit. This is signaled also before doing the full expansion in $q$ as the first term in (5) diverges at zero crossing. However, in effective potentials derived from the $T$-matrix these problems are not transparent as the lowest order coefficient is proportional to $a(B)$ (see (12)). Below we will discuss what kind of constraints this introduces on the applicability of the effective-range expansion near zero crossing. We note that similar issues were briefly discussed in a different context in [34] where an equivalent to (7) below was obtained.

Let us first consider the low- $q$ limit and compare the full $T$-matrix with the effective-range expansion as zero crossing is approached. Taking the low- $q$ limit of (4) at zero crossing where $\Delta B /\left(B-B_{0}\right)=1$, we find

$$
q \cot \delta(q) \longrightarrow \frac{-1}{a_{b g}}-\frac{\Delta \mu \Delta B}{\hbar^{2} q^{2} / m},
$$

which diverges as $q^{-2}$. Therefore the coefficients of the expansion in (5) must necessarily diverge in order to retain any hope of describing the low- $q$ behavior. Furthermore, since the expansion is an alternating series and therefore slowly converged, we also conclude that many terms must be retained for a fair approximation at very small but nonzero $q$. The same conclusion can be reached by considering the radius of convergence of (5), which we find by locating the pole in (4) at $\hbar^{2} q^{2} / m=\Delta \mu\left(B-B_{0}-\Delta B\right)$. This radius indeed goes to zero at zero crossing. We are thus forced to conclude that the effective-range expansion breaks down near zero crossing.
2.1. Effective Potential at Zero Crossing. Since the effectiverange expansion is insufficient we consider the full $T$-matrix in the low- $q$ limit at zero crossing. To lowest order we have

$$
T_{o o}\left(B=B_{0}+\Delta B\right)=-\frac{4 \pi \hbar^{2} a_{b g}}{m} \frac{\hbar^{2} q^{2}}{m \Delta \mu \Delta B}+O\left(q^{4}\right) .
$$

Using the expression for $r_{e 0}$, this can be written

$$
\frac{4 \pi \hbar^{2}}{m} \frac{a_{b g}^{2} r_{e 0}}{2} q^{2}
$$

Knowing the $T$-matrix at low $q$ we can now proceed to find an effective low- $q$ potential through the LippmannSchwinger equation:

$$
V=T-T G_{0} V
$$

where $G_{0}=\left(E-H_{0}+i \delta\right)^{-1}$ is the free space Green's function [35]. This equation can be solved for $T\left(q, q^{\prime}\right) \propto q^{2}+q^{\prime 2}$ (the symmetrized version of the full $T$-matrix) in an explicit cutoff approach $[29,35]$ and then be expanded to order $q^{2}$ for consistence with the input $T$-matrix. In the long-wavelength limit we can take the cut-off to zero [35] and for the on-shell effective potential we then obtain the obvious answer:

$$
V(q)=\frac{4 \pi \hbar^{2}}{m} \frac{a_{b g}^{2} r_{e 0}}{2} q^{2}
$$

in momentum space. The effective potential in real-space is now easily found by canonical substitution $(\mathbf{q} \rightarrow-i \nabla)$ and appropriate symmetrization [36]. We have

$$
V(\mathbf{r})=-\frac{4 \pi \hbar^{2}}{m} \frac{a_{b g}^{2} r_{e 0}}{2} \frac{1}{2}\left[\leftarrow_{\mathbf{r}}^{2} \delta(\mathbf{r})+\delta(\mathbf{r}) \vec{\nabla}_{\mathbf{r}}^{2}\right] .
$$

Notice that the Lippmann-Schwinger approach is nonperturbative as opposed to the perturbative energy shift method $[36,37]$.

2.2. Comparison to Effective-Range Expansion and EnergyShift Method. Away from zero crossing one can easily relate the effective-range expansion to an effective potential through the perturbative energy shift method $[18,25,26]$. To second order the $s$-wave effective potential is

$$
V(\mathbf{r})=\frac{4 \pi \hbar^{2} a}{m}\left[\delta(\mathbf{r})+\frac{g_{2}}{2}\left(\overleftarrow{\nabla}_{\mathbf{r}}^{2} \delta(\mathbf{r})+\delta(\mathbf{r}) \vec{\nabla}_{\mathbf{r}}^{2}\right)\right],
$$

where the first term is the effective interaction usually employed in mean-field theories of cold atoms [35]. In terms of $a$ and $r_{e}$, we have $g_{2}=a^{2} / 3-a r_{e} / 2[36,37]$ with the fielddependent $a=a(B)$ and $r_{e}=r_{e}(B)$.

At zero-crossing the first term in (12) vanishes and one might expect the second term to vanish as well. However, in the naive effective-range expansion of the two-channel model discussed above we saw that $r_{e}$ diverges as $a^{-2}$ and we therefore have

$$
\lim _{a \rightarrow 0} a g_{2}=-\frac{a_{b g}^{2} r_{e 0}}{2}
$$


In particular, if we for a moment ignore $q^{4}$ terms in the effective-range expansion, we recover exactly the same effective potential as in (11) at zero crossing. The finite limiting result in (13) shows that the potential in (12) is well defined as $a \rightarrow 0$, provided that appropriate regularization and renormalization are performed. Equation (12) thus applies equally well at resonance $(a \rightarrow \infty)$ where the gradient terms are small and at zero crossing where the lowest order delta function term is unimportant. It is thus a welldefined effective potential over the entire range of a Feshbach resonance.

We therefore see that even though the effective-range expansion has divergent coefficients at zero crossing, the lowest order does in fact give the same effective potential as the full $T$-matrix if we apply it naively. The effective-range expansion should thus be viewed as an asymptotic series. However, we cannot use the effective-range expansion to estimate the validity of the second-order effective potential since the radius of convergence goes to zero at zero crossing as discussed above.

The two-channel model in (1) compares well with a coupled-channel calculation [33] as shown in Figure 1. It also compares well to other scattering models $[38,39]$ that include finite-range effects. In fact, the model used here compares well with the analytical models of [38] when $a(B)$ and $r_{e}(B)$ have the field-dependence introduced above. This can be seen for instance in Figure 12 of [38], although a difference is that our $a(B)$ and $r_{e}(B)$ are parametrization and not taken from coupled-channels values as in [38] (our Figure 1 quantifies the difference which is largest on $r_{e}(B)$ ). However, here we are concerned with the behavior when $a(B) \rightarrow 0$ in the context of Feshbach resonances which is not addressed in $[38,39]$. We note that the resonance models of [31] and the two-channel and resonance models in [32] are very similar to the model employed here, but again those references do not consider the specific problems arising when $a(B) \rightarrow 0$. In addition and in contrast to previous discussions, here we construct appropriate zerorange pseudo potentials that work around zero crossing.

\section{Relation to Experiments}

Above we only retained terms of order $q^{2}$ in the full $T$-matrix. We now estimate the energy regime in which this expression is valid. Demanding that the $q^{4}$ term be smaller than the $q^{2}$ term gives the criterion:

$$
\frac{\hbar^{2} q^{2}}{m} \ll \frac{\hbar^{2}}{m\left|a_{b g} r_{e 0}\right|} .
$$

We relate this condition to recent experiments with bosonic condensates of ${ }^{39} \mathrm{~K}$ working around zero crossing [3]. The resonance used there is very broad $(\Delta B=-52 \mathrm{G})$ with $a_{b g}=-29 a_{0}$ and $r_{e 0}=-58 a_{0}$ ( $a_{0}$ is the Bohr radius). The right-hand side of (14) is $2.3 \cdot 10^{-7} \mathrm{eV}$, corresponding to a temperature of about $3 \mathrm{mK}$. Since the experiments are performed at much lower temperatures the approximation above is certainly valid. However, as $a_{b g}$ and particularly $r_{e 0}$ are small, the front factor in (11) is also small. The relevant scale of comparison is the outer trap parameter $b$ [9] which is typically of order $1 \mu \mathrm{m}$, yielding a vanishing ratio $\left|a_{b g}^{2} r_{e 0}\right| / b^{3} \sim 10^{-9}$. For broad Feshbach resonances the higher order interactions can thus be safely ignored. For very narrow resonances the situation potentially changes as $r_{e 0}$ can be very large and make the potential in (11) important. As an example, we consider the narrow resonance in ${ }^{39} \mathrm{~K}$ at $B_{0}=25.85 \mathrm{G}$ with $\Delta B=0.47 \mathrm{G}, a_{b g}=-33 a_{0}$, and $r_{e 0}=$ $-5687 a_{0}$ [5]. The right-hand side of $(14)$ is now $2 \cdot 10^{-9} \mathrm{eV}$, corresponding to $24 \mu \mathrm{K}$. This is again much higher than experimental temperatures. A more careful argument can be made from the energy per particle of the noncondensed cloud. Ignoring the trap, we have $E / N=0.770 k_{B} T_{c}\left(T / T_{c}\right)^{5 / 2}$ ( $T_{c}$ is the critical temperature) [35]. For a sample of $3 \cdot 10^{4}$ a critical temperature of $100 \mathrm{nK}$ was reported in [6]. Using this $T_{c}$ we find that $T \ll 900 \mathrm{nK}$ for (14) to hold. Again this is within the experimental regime. The effective potential approach should therefore be applicable around zero crossing for narrow resonances. However, even with this narrow resonance we find $\left|a_{b g}^{2} r_{e 0}\right| / b^{3} \sim 10^{-7}$, and the effect is still completely negligible.

In order to increase the relevance of the higher order term, we now consider some very narrow resonances that have been found in ${ }^{87} \mathrm{Rb}$. In particular, the resonance at $B_{0}=$ $9.13 \mathrm{G}[40]$ which was recently utilized in nonlinear atom interferometry [41]. We have $\Delta B=0.015 \mathrm{G}, a_{b g}=99.8 a_{0}$, and $\Delta \mu=2.00 \mu_{B}[42]$, which gives $r_{e 0}=-19.8 \cdot 10^{3} a_{0}$ and a ratio $\left|a_{b g}^{2} r_{e 0}\right| / b^{3}=2.92 \cdot 10^{-5}(1 \mu \mathrm{m} / b)^{3}$. A trap length of $b \sim 0.5 \mu \mathrm{m}$ as used in [41] would thus yield $10^{-4}$ and demonstrates that higher order corrections can safely be neglected. For a ratio of 1 we need $b \sim 0.03 \mu \mathrm{m}$ which is unrealistically small in current traps or optical lattices. However, a resonance of width $\Delta B=0.0004 \mathrm{G}$ is known in the same system at $B_{0}=406.2 \mathrm{G}$ [43] with $a_{b g}=100 a_{0}$ and $\Delta \mu=2.01 \mu_{B}$ [42]. In this case we find $r_{e 0}=-7.4 \cdot 10^{5} a_{0}$ and a much more favorable ratio of $\left|a_{b g}^{2} r_{e 0}\right| / b^{3}=0.001(1 \mu \mathrm{m} / b)^{3}$. Here we see that a ratio of 1 is achieved already for $b \sim$ $0.1 \mu \mathrm{m}$ which not far off from tight traps or optical lattice dimensions. In terms of temperature we still have to be in the ultralow regime of $T \lesssim 30 \mathrm{nK}$ according to (14) for the latter resonance.

Consider now a fermionic two-component system where $s$-wave interactions are dominant. Since we have $r_{e 0}<0$ for all Feshbach resonances [42], the effective potential in (10) is attractive, and the system could potentially be unstable toward a paired state or become unstable to collapse above a critical particle number. For simplicity we will use the semiclassical Thomas-Fermi approach to describe a gas with equal population of the two components and estimate the critical particle number. Assuming an isotropic trapping potential with length scale $b=\sqrt{\hbar / m \omega}$ where $\omega$ is the trap frequency, the ground-state density, $\rho(\mathbf{x})$, can be found by minimization and satisfies

$$
\left[\frac{\mu}{\hbar \omega}-\frac{1}{2}\left(\frac{\mathbf{x}}{b}\right)^{2}\right]=\frac{1}{2}\left(k_{F}(\mathbf{x}) b\right)^{2}-\frac{4}{30 \pi} \alpha\left(k_{F}(\mathbf{x}) b\right)^{5},
$$

where $\rho(\mathbf{x})=k_{F}(\mathbf{x}) / 6 \pi^{2}$ and $\alpha=a_{b g}^{2}\left|r_{e 0}\right| / b^{3}$. The maximum allowed momentum and chemical potential, $\mu$, is found by 
solving for the turning point of the right-hand side of (15) which gives

$$
k_{\max } b=\left[\frac{3 \pi}{2 \alpha}\right]^{1 / 3}, \quad \mu_{\max }=\frac{3}{10} \hbar \omega\left(k_{\max } b\right)^{2} .
$$

We can now compare this $k_{\max }$ to the value obtained from the noninteracting density within the Thomas-Fermi approximation at the center of the trap. In terms of the number of particles in each component, $N$, at the center of the trap we have $k_{F}(0) b \approx 1.906 N^{1 / 6}$ [35]. By equating these two expression we obtain an estimate for the critical number of particles, $N_{\max }$. Inserting the relevant units, we have

$$
N_{\max }=2 \cdot 10^{25}\left(\frac{a_{0}}{a_{b g}}\right)^{4}\left(\frac{a_{0}}{r_{e 0}}\right)^{2}\left(\frac{b}{1 \mu \mathrm{m}}\right)^{6},
$$

where $a_{0}$ is the Bohr radius. We note that the scaling $N_{\max } \propto$ $\alpha^{-2}$ can also be obtained by considering the point at which the monopole mode becomes unstable.

Typical numbers for common fermionic species ${ }^{6} \mathrm{Li}$ or ${ }^{40} \mathrm{~K}$ in the lowest hyperfine states [42] lead to $N_{\max } \sim 10^{12}$ for $b=1 \mu \mathrm{m}$. This is of course a huge number and experiments are well within this limit. Even if one reduced the trap length by a factor of ten and made the presumably unrealistic assumption that the particle number remains the same we still have $N \ll N_{\max }$. The reason is that the $s$-wave Feshbach resonances utilized in the two-component gases are generally broad in order to study the universal regime. If we consider the narrow resonance at $B_{0}=543.25 \mathrm{G}$ in ${ }^{6} \mathrm{Li}$ [44] with $\Delta B=0.1 \mathrm{G}, a_{b g}=60 a_{0}$, and $\Delta \mu=2.00 \mu_{B}$ [42], we have $N_{\max } \sim 2 \cdot 10^{13}(b / 1 \mu \mathrm{m})^{6}$. This is somewhat better but we still need $b \sim 0.06 \mu \mathrm{m}$ to get to an experimentally relevant $N_{\max } \sim 10^{6}$. We have to conclude that higher order $s$-wave interactions are highly unlikely to be observable through monopole instabilities. In light of this it seems better to consider $p$-wave resonances which are much more narrow in general. However, also here extremely small trap sizes appear necessary [45].

The instability toward Cooper pairing around zero crossing can also be estimated in simple terms. In general the critical temperature is $T_{c} \sim T_{F} \exp \left(-1 / N_{0}|U|\right)$, where $N_{0}=$ $m k_{F}(0) / 2 \pi^{2} \hbar^{2}$ is the density of states at the Fermi energy in the trap center and $U<0$ is a measure of the attraction. For the latter we use the effective potential in momentum space from (10) and make the assumption that $q \sim k_{F}(0)$. Using the expression for $k_{F}(0)$ in terms of $N$ above, we find

$$
\frac{1}{N_{0}|U|}=\frac{1.5 \cdot 10^{12}}{\sqrt{N}}\left(\frac{b}{1 \mu \mathrm{m}}\right)^{3}\left(\frac{a_{0}}{a_{b g}}\right)^{2} \frac{a_{0}}{\left|r_{e 0}\right|} .
$$

For broad resonances in ${ }^{6} \mathrm{Li}$ or ${ }^{40} \mathrm{~K}$ this exponent is of order $10^{3}$ and $T_{c}$ is thus vanishingly small. However, the scaling with trap size can help and if we imagine reducing to $b=$ $0.1 \mu \mathrm{m}$, we find $T_{c} \lesssim 0.5 T_{F}$ for $N=10^{6}$ atoms. For the narrow resonance in ${ }^{6} \mathrm{Li}$ discussed above, we find that $T_{c} \sim$ $0.5 T_{F}$ with $N=10^{6}$ can be achieved for $b \sim 0.5 \mu \mathrm{m}$ and $T_{c} \sim 0.1 T_{F}$ for $N=10^{5}$. Thus there may be a possibility to reach the pairing instability near zero crossing if high particle numbers can be cooled in tight traps and narrow resonances are used.

While the suboptical wavelength trapping sizes needed for the above effects to be large are not achievable with typical optical or magnetic traps or optical lattice setups, they could potentially be reached via hybrid setups where atoms are trapped near a surface. Inspired by surface plasmon subwavelength optics [46], nanoscale trapping for neutral atoms has been studied $[47,48]$, and micropotential traps with width less than 100 nanometer $(<0.1 \mu \mathrm{m})$ are within reach [49]. In these very tightly confined systems, it is very likely that finite-range effects could be enhanced. Devices that provide an interface between atoms and solidstate systems are under intense study at the moment, and our considerations here imply that finite-range corrections should be considered when the scattering length is tuned close to zero.

3.1. Dipole-Dipole Interactions. The discussion above ignores the dipole-dipole interaction discussed in the introduction which will compete against the higher order effective potential from the Feshbach resonance. A simple estimate can be made along the lines of the discussion in [35]. The external trapping potential is the characteristic scale of spatial variations and we thus find a ratio, $r$, of magnetic dipoledipole, $U_{\mathrm{md}}$, to higher-order $s$-wave zero-range interaction strength, $U_{2}$, which can be written as

$$
r=\frac{U_{\mathrm{md}}}{U_{2}}=\frac{a_{0} b^{2}}{a_{b g}^{2}\left|r_{e 0}\right|}=35.7\left[\frac{b}{1 \mu \mathrm{m}}\right]^{2}\left[\frac{100 a_{0}}{a_{b g}}\right]^{2} \frac{1000 a_{0}}{\left|r_{e 0}\right|} .
$$

For $r<1$ the higher order interaction term will therefore dominate the magnetic dipole term. For the case of narrow resonances in ${ }^{87} \mathrm{Rb}$ discussed above we find $r \sim$ $0.11(b / 1 \mu \mathrm{m})^{2}$ for the resonance at $B_{0}=9.13 \mathrm{G}$ and $r \sim$ $0.05(b / 1 \mu \mathrm{m})^{2}$ for the one at $B_{0}=406.2 \mathrm{G}$. For the narrow resonance in ${ }^{6} \mathrm{Li}$ at $B_{0}=543.25 \mathrm{G}$ we find $r \sim 1.4(b / 1 \mu \mathrm{m})^{2}$. These ratios clearly indicate that magnetic dipole-dipole interactions can be suppressed relative to higher order zerorange terms for narrow Feshbach resonances and standard trap sizes. This dominance becomes even stronger for the tight traps needed for the realization of the effects discussed above, and we thus conclude that interference of the magnetic dipole-dipole term is not a major concern.

\section{Conclusions and Outlook}

In this paper we have discussed the effective potential around a Feshbach resonances as the scattering length is tuned to zero and finite-range corrections become important. We showed that the effective-range expansion is badly behaved and the effective potential most be defined from the $T$ matrix. We have demonstrated that the low momenta effective potential obtained from the full $T$-matrix agrees with one obtained naively from the effective-range expansion when the scattering length goes to zero. Thus even though the effective-range expansion has divergent coefficients at zerocrossing the first terms of the associated effective potential 
yield consistent results. We then estimated the effects of the terms on different condensates. Since the effective potential at zero crossing is attractive it may induce various instabilities which we considered for the case of a two-component Fermi gas under harmonic confinement.

For the broad Feshbach resonances used in current experiments the effective potential discussed here are negligible and the dipole-dipole interaction dominates completely at zero crossing. However, for narrow resonances in very tightly confined systems some of the effects might be detectable. In particular, future generations of microtraps with suboptical wavelength trap sizes using surface plasmons could be small enough to make finite-range effects important. The competing dipole interaction is small for narrow resonances in tight confinement. However, it is conceivable that effects of spherically symmetric higher order terms could be separated from dipolar effects which change with system geometry [4].

Small trapped Fermi systems have recently become an experimental reality with particle numbers ranging from two to ten [50]. For two atomic fermions with different internal states, the system turns out to be well described by the analytic zero-range model of Busch et al. [51-56], and similarly for three fermions [57, 58]. Effective-range corrections to these results have also been studied [59-62]. Mesoscopic Fermi systems (less than about 50 particles) have been studied in harmonic traps using a number of numerical methods, [63-79] with particular emphasis on the unitary regime where the scattering length diverges. It would be interesting to investigate the situation also around zerocrossing of a narrow resonance where the effective range is sizable. A preliminary study along this line for three bosons is discussed in [80].

Another interesting direction of future work is the study of the contact introduced by Tan [81-90] to describe the universal behavior of strongly interacting quantum gases at a broad resonance where the range corrections are negligible, for instance through the tail of the momentum distribution which is predicted to behave as $C / k^{4}$, where $C$ is the contact and $k$ the momentum of a single particle. The relations found by Tan $[81,82]$ have subsequently been confirmed experimentally in three dimensions [91-93]. While the contact originally pertains to two-body correlations, signatures of three-body physics in momentum distributions have also been studied both theoretically [86, 94-98] and experimentally [99]. While a few studies have considered the universal behavior when including the effective range term $[100,101]$, it would be very interesting to consider the regime around zero crossing for a narrow resonance where the background effective range parameter.

\section{Acknowledgments}

The author would like to thank Martin Thøgersen for very fruitful collaborations. Correspondence with Georg Bruun about two-channel models is highly appreciated. The author is grateful to Nicolai Nygaard for discussions and for producing Figure 1. The author acknowledges the hospitality of the Niels Bohr Institute, Blegdamsvej 17, 2100
Copenhagen $\varnothing$, Denmark. This work was supported by the Villum Kann Rasmussen foundation.

\section{References}

[1] I. Bloch, J. Dalibard, and W. Zwerger, "Many-body physics with ultracold gases," Reviews of Modern Physics, vol. 80, no. 3, pp. 885-964, 2008.

[2] C. Chin, R. Grimm, P. Julienne, and E. Tiesinga, "Feshbach resonances in ultracold gases," Reviews of Modern Physics, vol. 82, no. 2, pp. 1225-1286, 2010.

[3] M. Fattori, C. D’Errico, G. Roati et al., "Atom interferometry with a weakly interacting bose-Einstein condensate," Physical Review Letters, vol. 100, no. 8, Article ID 080405, 4 pages, 2008.

[4] M. Fattori, G. Roati, B. Deissler et al., "Magnetic dipolar interaction in a Bose-Einstein condensate atomic interferometer," Physical Review Letters, vol. 101, no. 19, Article ID 190405, 4 pages, 2008.

[5] C. D'Errico, M. Zaccanti, M. Fattori et al., "Feshbach resonances in ultracold ${ }^{39} \mathrm{~K}$," New Journal of Physics, vol. 9, article 223, 2007.

[6] G. Roati, M. Zaccanti, C. D'Errico et al., " ${ }^{39} \mathrm{~K}$ bose-Einstein condensate with tunable interactions," Physical Review Letters, vol. 99, no. 1, Article ID 010403, 4 pages, 2007.

[7] S. E. Pollack, D. Dries, M. Junker, Y. P. Chen, T. A. Corcovilos, and R. G. Hulet, "Extreme tunability of interactions in a ${ }^{7} \mathrm{Li}$ Bose-Einstein condensate," Physical Review Letters, vol. 102, no. 9, Article ID 090402, 4 pages, 2009.

[8] H. Fu, Y. Wang, and B. Gao, "Beyond the Fermi pseudopotential: a modified Gross-Pitaevskii equation," Physical Review, vol. 67, no. 5, Article ID 053612, 6 pages, 2003.

[9] N. T. Zinner and M. Thøgersen, "Stability of a Bose-Einstein condensate with higher-order interactions near a Feshbach resonance," Physical Review, vol. 80, no. 2, Article ID 023607, 4 pages, 2009.

[10] M. Thøgersen, N. T. Zinner, and A. S. Jensen, "ThomasFermi approximation for a condensate with higher-order interactions," Physical Review A, vol. 80, no. 4, Article ID 043625, 8 pages, 2009.

[11] E. Stoner, "LXXX. Atomic moments in ferromagnetic metals and alloys with non-ferromagnetic elements," Philosophical Magazine, vol. 15, no. 101, pp. 1018-1034, 1933.

[12] M. Houbiers, R. Ferwerda, H. T. C. Stoof, W. I. McAlexander, C. A. Sackett, and R. G. Hulet, "Superfluid stateof atomic ${ }^{6} \mathrm{Li}$ in a magnetic trap," Physical Review A, vol. 56, no. 6, pp. 4864-4878, 1997.

[13] Y. Zhang and S. Das Sarma, "Exchange instabilities in electron systems: bloch versus Stoner ferromagnetism," Physical Review B, vol. 72, no. 11, Article ID 115317, 9 pages, 2005.

[14] R. A. Duine and A. H. MacDonald, "Itinerant ferromagnetism in an ultracold atom Fermi gas," Physical Review Letters, vol. 95, no. 23, Article ID 230403, 4 pages, 2005.

[15] G. J. Conduit and B. D. Simons, "Itinerant ferromagnetism in an atomic Fermi gas: influence of population imbalance," Physical Review A, vol. 79, no. 5, Article ID 053606, 9 pages, 2009.

[16] J. Conduit, A. G. Green, and B. D. Simons, "Inhomogeneous phase formation on the border of itinerant ferromagnetism," Physical Review Letters, vol. 103, no. 20, Article ID 207201, 4 pages, 2009.

[17] G. J. Conduit and B. D. Simons, "Repulsive atomic gas in a harmonic trap on the border of itinerant ferromagnetism," 
Physical Review Letters, vol. 103, no. 20, Article ID 200403, 4 pages, 2009.

[18] L. J. Leblanc, J. H. Thywissen, A. A. Burkov, and A. Paramekanti, "Repulsive Fermi gas in a harmonic trap: ferromagnetism and spin textures," Physical Review A, vol. 80, no. 1, Article ID 013607, 2009.

[19] S. Zhang, H.-H. Hung, and C. Wu, "Proposed realization of itinerant ferromagnetism in optical lattices," Physical Review, vol. 82, no. 5, Article ID 053618, 5 pages, 2010.

[20] G. B. Jo, Y. R. Lee, J. H. Choi et al., "Itinerant ferromagnetism in a fermi gas of ultracold atoms," Science, vol. 325, no. 5947, pp. 1521-1524, 2009.

[21] H. Zhai, "Correlated versus ferromagnetic state in repulsively interacting two-component Fermi gases," Physical Review A, vol. 80, no. 5, Article ID 051605, 4 pages, 2009.

[22] X. Cui and H. Zhai, "Stability of a fully magnetized ferromagnetic state in repulsively interacting ultracold Fermi gases," Physical Review A, vol. 81, no. 4, Article ID 041602, 4 pages, 2010.

[23] D. Pekker, M. Babadi, R. Sensarma et al., "Competition between pairing and ferromagnetic instabilities in ultracold Fermi gases near Feshbach resonances," Physical Review Letters, vol. 106, no. 5, Article ID 050402, 4 pages, 2011.

[24] V. B. Shenoy and T.-L. Ho, "Nature and properties of a repulsive Fermi gas in the upper branch of the energy spectrum," Physical Review Letters, vol. 107, no. 21, Article ID 210401, 5 pages, 2011.

[25] C. Sanner, E. J. Su, W. Huang, A. Keshet, J. Gillen, and W. Ketterle, "Correlations and pair formation in a repulsively interacting Fermi gas," Physical Review Letters, vol. 108, no. 24, Article ID 240404, 5 pages, 2012.

[26] C. Kohstall, M. Zaccanti, M. Jag et al., "Metastability and coherence of repulsive polarons in a strongly interacting Fermi mixture," Nature, vol. 485, pp. 615-618, 2012.

[27] T. H. R. Skyrme, "CVII. The nuclear surface," Philosophical Magazine, vol. 1, no. 11, pp. 1043-1054, 1956.

[28] T. H. R. Skyrme, "The effective nuclear potential," Nuclear Physics, vol. 9, no. 4, pp. 615-634, 1959.

[29] D. R. Phillips, S. R. Beane, and T. D. Cohen, "Nonperturbative regularization and renormalization: simple examples from nonrelativistic quantum mechanics," Annals of Physics, vol. 263, no. 2, pp. 255-275, 1998.

[30] G. M. Bruun, A. D. Jackson, and E. E. Kolomeitsev, "Multichannel scattering and Feshbach resonances: effective theory, phenomenology, and many-body effects," Physical Review A, vol. 71, no. 5, Article ID 052713, 10 pages, 2005.

[31] S. J. J. M. F. Kokkelmans, J. N. Milstein, M. L. Chiofalo, R. Walser, and M. J. Holland, "Resonance superfluidity: rsenormalization of resonance scattering theory," Physical Review A, vol. 65, no. 5, Article ID 536171, 4 pages, 2002.

[32] E. Braaten, M. Kusunoki, and D. Zhang, "Scattering models for ultracold atoms," Annals of Physics, vol. 323, no. 7, pp. 1770-1815, 2008.

[33] N. Nygaard, B. I. Schneider, and P. S. Julienne, "Two-channel R-matrix analysis of magnetic-field-induced Feshbach resonances," Physical Review A, vol. 73, no. 4, Article ID 042705, 10 pages, 2006.

[34] P. Massignan and Y. Castin, "Three-dimensional strong localization of matter waves by scattering from atoms in a lattice with a confinement-induced resonance," Physical Review A, vol. 74, no. 1, Article ID 013616, 2006.

[35] C. J. Pethick and H. Smith, Bose-Einstein Condensation in Dilute Gases, Cambridge University Press, Cambridge, Mass, USA, 2002.
[36] R. Roth and H. Feldmeier, "Effective $s$ - and p-wave contact interactions in trapped degenerate Fermi gases," Physical Review A, vol. 64, no. 4, Article ID 043603, 17 pages, 2001.

[37] A. Collin, P. Massignan, and C. J. Pethick, "Energydependent effective interactions for dilute many-body systems," Physical Review A, vol. 75, no. 1, Article ID 013615, 2007.

[38] B. Marcelis, E. G. M. van Kempen, B. J. Verhaar, and S. J. J. M. F. Kokkelmans, "Feshbach resonances with large background scattering length: interplay with open-channel resonances," Physical Review A, vol. 70, no. 1, Article ID 012701, 15 pages, 2004.

[39] B. Marcelis and S. Kokkelmans, "Fermionic superfluidity with positive scattering length," Physical Review A, vol. 74, no. 2, Article ID 023606, 2006.

[40] A. Widera, O. Mandel, M. Greiner, S. Kreim, T. W. Hänsch, and I. Bloch, "Entanglement interferometry for precision measurement of atomic scattering properties," Physical Review Letters, vol. 92, no. 16, Article ID 160406, 2004.

[41] C. Gross, T. Zibold, E. Nicklas, J. Estève, and M. K. Oberthaler, "Nonlinear atom interferometer surpasses classical precision limit," Nature, vol. 464, no. 7292, pp. 11651169,2010

[42] C. Chin, R. Grimm, P. Julienne, and E. Tiesinga, "Feshbach resonances in ultracold gases," Reviews of Modern Physics, vol. 82, no. 2, pp. 1225-1286, 2010.

[43] A. Marte, T. Volz, J. Schuster et al., "Feshbach resonances in rubidium 87: precision measurement and analysis," Physical Review Letters, vol. 89, no. 28, Article ID 283202, 4 pages, 2002.

[44] K. E. Strecker, G. B. Partridge, and R. G. Hulet, "Conversion of an atomic Fermi gas to a long-lived molecular bose gas," Physical Review Letters, vol. 91, no. 8, Article ID 080406, 4 pages, 2003.

[45] N. T. Zinner, "Stability of a fully polarized ultracold Fermi gas near zero-crossing of a p-wave Feshbach resonance," European Physical Journal D, vol. 57, no. 2, pp. 235-240, 2010.

[46] W. L. Barnes, A. Dereux, and T. W. Ebbesen, "Surface plasmon subwavelength optics," Nature, vol. 424, no. 6950, pp. 824-830, 2003.

[47] B. Murphy and L. V. Hau, "Electro-optical nanotraps for neutral atoms," Physical Review Letters, vol. 102, no. 3, Article ID 033003, 4 pages, 2009.

[48] D. E. Chang, J. D. Thompson, H. Park et al., “Trapping and manipulation of isolated atoms using nanoscale plasmonic structures," Physical Review Letters, vol. 103, no. 12, Article ID 123004, 4 pages, 2009.

[49] C. Stehle, H. Bender, C. Zimmermann, D. Kern, M. Fleischer, and S. Slama, "Plasmonically tailored micropotentials for ultracold atoms," Nature Photonics, vol. 5, no. 8, pp. 494-498, 2011.

[50] F. Serwane, G. Zürn, T. Lompe, T. B. Ottenstein, A. N. Wenz, and S. Jochim, "Deterministic preparation of a tunable fewfermion system," Science, vol. 332, no. 6027, pp. 336-338, 2011.

[51] T. Busch, B. G. Englert, K. Rzazewski, and M. Wilkens, "Two cold atoms in a harmonic trap," Foundations of Physics, vol. 28, no. 4, pp. 549-559, 1998.

[52] T. Stöferle, H. Moritz, K. Günter, M. Köhl, and T. Esslinger, "Molecules of fermionic atoms in an optical lattice," Physical Review Letters, vol. 96, no. 3, Article ID 030401, 4 pages, 2006.

[53] T. Volz, N. Syassen, D. M. Bauer, E. Hansis, S. Dürr, and G. Rempe, "Preparation of a quantum state with one molecule 
at each site of an optical lattice," Nature Physics, vol. 2, no. 10, pp. 692-695, 2006.

[54] G. Thalhammer, K. Winkler, F. Lang, S. Schmid, R. Grimm, and J. Hecker Denschlag, "Long-lived Feshbach molecules in a three-dimensional optical lattice," Physical Review Letters, vol. 96, no. 5, Article ID 050402, 4 pages, 2006.

[55] C. Ospelkaus, S. Ospelkaus, L. Humbert, P. Ernst, K. Sengstock, and K. Bongs, "Ultracold heteronuclear molecules in a 3D optical lattice," Physical Review Letters, vol. 97, no. 12, Article ID 120402, 4 pages, 2006.

[56] D. Blume and C. H. Greene, "Fermi pseudopotential approximation: two particles under external confinement," Physical Review A, vol. 65, no. 4, Article ID 043613, 6 pages, 2002.

[57] F. Werner and Y. Castin, "Unitary quantum three-body problem in a. Harmonic trap," Physical Review Letters, vol. 97, Article ID 150401, 2006.

[58] F. Werner and Y. Castin, "Unitary gas in an isotropic harmonic trap: symmetry properties and applications," Physical Review A, vol. 74, no. 5, Article ID 053604, 2006.

[59] Z. Idziaszek and T. Calarco, "Analytical solutions for the dynamics of two trapped interacting ultracold atoms," Physical Review A, vol. 74, no. 2, Article ID 022712, 2006.

[60] A. Suzuki, Y. Liang, and R. K. Bhaduri, "Two-atom energy spectrum in a harmonic trap near a Feshbach resonance at higher partial waves," Physical Review A, vol. 80, no. 3, Article ID 033601, 6 pages, 2009.

[61] S. G. Peng, S. Q. Li, P. D. Drummond, and X. J. Liu, "Hightemperature thermodynamics of strongly interacting s-wave and p-wave Fermi gases in a harmonic trap," Physical Review A, vol. 83, no. 6, Article ID 063618, 10 pages, 2011.

[62] N. T. Zinner, "Universal two-body spectra of ultracold harmonically trapped atoms in two and three dimensions," Journal of Physics A, vol. 45, no. 20, Article ID 205302, 2012.

[63] J. L. DuBois and H. R. Glyde, "Bose-Einstein condensation in trapped bosons: a variational Monte Carlo analysis," Physical Review A, vol. 63, no. 2, Article ID 023602, 2001.

[64] J. Carlson, S. Y. Chang, V. R. Pandharipande, and K. E. Schmidt, "Superfluid Fermi gases with large scattering length," Physical Review Letters, vol. 91, no. 5, Article ID 050401, 4 pages, 2003.

[65] S. Y. Chang and G. F. Bertsch, "Unitary Fermi gas in a harmonic trap," Physical Review A, vol. 76, no. 2, Article ID 021603, 2007.

[66] D. Blume, J. Von Stecher, and C. H. Greene, "Universal properties of a trapped two-component fermi gas at unitarity," Physical Review Letters, vol. 99, no. 23, Article ID 233201, 2007.

[67] M. Thøgersen, D. V. Fedorov, and A. S. Jensen, "Trapped Bose gases with large positive scattering length," vol. 79, no. 4, Article ID 40002, 6 pages, 2007.

[68] J. von Stecher, C. H. Greene, and D. Blume, "Energetics and structural properties of trapped two-component Fermi gases," Physical Review A, vol. 77, no. 4, Article ID 043619, 20 pages, 2008.

[69] D. Lee, "Lattice simulations for few- and many-body systems," Progress in Particle and Nuclear Physics, vol. 63, no. 1, pp. 117-154, 2009.

[70] W. C. Haxton and T. Luu, "Perturbative effective theory in an oscillator basis?” Physical Review Letters, vol. 89, no. 18, Article ID 182503, 4 pages, 2002.

[71] I. Stetcu, B. R. Barrett, U. van Kolck, and J. P. Vary, "Effective theory for trapped few-fermion systems," Physical Review A, vol. 76, no. 6, Article ID 063613, 7 pages, 2007.
[72] Y. Alhassid, G. F. Bertsch, and L. Fang, "New effective interaction for the trapped fermi gas," Physical Review Letters, vol. 100, no. 23, Article ID 230401, 2008.

[73] N. T. Zinner, K. M. Mølmer, C. Özen, D. J. Dean, and K. Langanke, "Shell-model Monte Carlo simulations of the BCS-BEC crossover in few-fermion systems," Physical Review A, vol. 80, no. 1, Article ID 013613, 5 pages, 2009.

[74] I. Stetcu, J. Rotureau, B. R. Barrett, and U. van Kolck, "An effective field theory approach to two trapped particles," Annals of Physics, vol. 325, no. 8, pp. 1644-1666, 2010.

[75] T. Luu, M. J. Savage, A. Schwenk, and J. P. Vary, "Nucleonnucleon scattering in a harmonic potential," Physical Review C, vol. 82, no. 3, Article ID 034003, 2010.

[76] J. Rotureau, I. Stetcu, B. R. Barrett, M. C. Birse, and U. Van Kolck, "Three and four harmonically trapped particles in an effective-field-theory framework," Physical Review A, vol. 82, no. 3, Article ID 032711, 2010.

[77] J. R. Armstrong, N. T. Zinner, D. V. Fedorov, and A. S. Jensen, "Analytic harmonic approach to the N-body problem," Journal of Physics B, vol. 44, no. 5, Article ID 055303, 2011.

[78] J. R. Armstrong, N. T. Zinner, D. V. Fedorov, and A. S. Jensen, "Quantum statistics and thermodynamics in the harmonic approximation," Physical Review E, vol. 85, no. 2, Article ID 021117, 10 pages, 2012.

[79] J. R. Armstrong, N. T. Zinner, D. V. Fedorov, and A. S. Jensen, "Virial expansion coefficients in the harmonicapproximation," http://arxiv.org/abs/1205.2574.

[80] N. T. Zinner, "Efimov trimers near the zero-crossing of a Feshbach resonance," http://arxiv.org/abs/1112.6358.

[81] S. Tan, "Energetics of a strongly correlated Fermi gas," Annals of Physics, vol. 323, no. 12, pp. 2952-2970, 2008.

[82] S. Tan, "Large momentum part of a strongly correlated Fermi gas," Annals of Physics, vol. 323, no. 12, pp. 2971-2986, 2008.

[83] E. Braaten and L. Platter, "Exact relations for a strongly interacting fermi gas from the operator product expansion," Physical Review Letters, vol. 100, no. 20, Article ID 205301, 2008.

[84] S. Zhang and A. J. Leggett, "Universal properties of the ultracold Fermi gas," Physical Review A, vol. 79, no. 2, Article ID 023601, 2009.

[85] R. Combescot, F. Alzetto, and X. Leyronas, "Particle distribution tail and related energy formula," Physical Review A, vol. 79, no. 5, Article ID 053640, 2009.

[86] F. Werner and Y. Castin, "Exact relations for quantummechanical few-body and many-body problems with short-range interactions in two and three dimensions," http://arxiv.org/abs/1001.0774.

[87] M. Barth and W. Zwerger, "Tan relations in one dimension," Annals of Physics, vol. 326, no. 10, pp. 2544-2565, 2011.

[88] M. Valiente, N. T. Zinner, and K. M. Mølmer, "Universal relations for the two-dimensional spin-1/2 Fermi gas with contact interactions," Physical Review A, vol. 84, no. 6, Article ID 063626, 4 pages, 2011.

[89] M. Valiente, "Tan's distributions and Fermi-Huang pseudopotential in momentum space," Physical Review A, vol. 85, no. 1, Article ID 014701, 4 pages, 2012.

[90] M. Valiente, N. T. Zinner, and K. M. Mølmer, "Universal properties of Fermi gases in arbitrary dimensions," http://arxiv.org/abs/1205.6388.

[91] J. T. Stewart, J. P. Gaebler, T. E. Drake, and D. S. Jin, "Rification of universal relations in a strongly interacting fermi gas," Physical Review Letters, vol. 104, no. 23, Article ID 235301, 2010. 
[92] E. D. Kuhnle, H. Hu, X. J. Liu et al., "Universal behavior of pair correlations in a strongly interacting fermi gas," Physical Review Letters, vol. 105, no. 7, Article ID 070402, 2010.

[93] E. D. Kuhnle, S. Hoinka, P. Dyke, H. Hu, P. Hannaford, and C. J. Vale, "Temperature dependence of the universal contact parameter in a unitary Fermi gas," Physical Review Letters, vol. 106, no. 17, Article ID 170402, 2011.

[94] E. Braaten, D. Kang, and L. Platter, "Universal relations for identical bosons from three-body physics," Physical Review Letters, vol. 106, no. 15, Article ID 153005, 2011.

[95] Y. Castin and F. Werner, "Single-particle momentum distribution of an Efimov trimer," Physical Review A, vol. 83, no. 6, Article ID 063614, 2011.

[96] K. Helfrich and H. W. Hammer, "Resonant three-body physics in two spatial dimensions," Physical Review A, vol. 83, no. 5, Article ID 052703, 7 pages, 2011.

[97] F. F. Bellotti, T. Frederico, M. T. Yamashita, D. V. Fedorov, A. S. Jensen, and N. T. Zinner, "Scaling and universality in two dimensions: three-body bound states with short-ranged interactions," Journal of Physics B, vol. 44, no. 20, Article ID 205302, 2011.

[98] F. F. Bellotti, T. Frederico, M. T. Yamashita, D. V. Fedorov, A. S. Jensen, and N. T. Zinner, "Supercircle description of universal three-body states in two dimensions," Physical Review A, vol. 85, no. 2, Article ID 025601, 5 pages, 2012.

[99] R. J. Wild, P. Makotyn, J. M. Pino, E. A. Cornell, and D. S. Jin, "Measurements of Tan's contact in an atomic bose-Einstein condensate," Physical Review Letters, vol. 108, no. 14, Article ID 145305, 5 pages, 2012.

[100] E. Braaten, D. Kang, and L. Platter, "Universal relations for a strongly interacting Fermi gas near a Feshbach resonance," Physical Review A, vol. 78, no. 5, Article ID 053606, 2008.

[101] F. Werner, "Virial theorems for trapped cold atoms," Physical Review A, vol. 78, no. 2, Article ID 025601, 4 pages, 2008. 

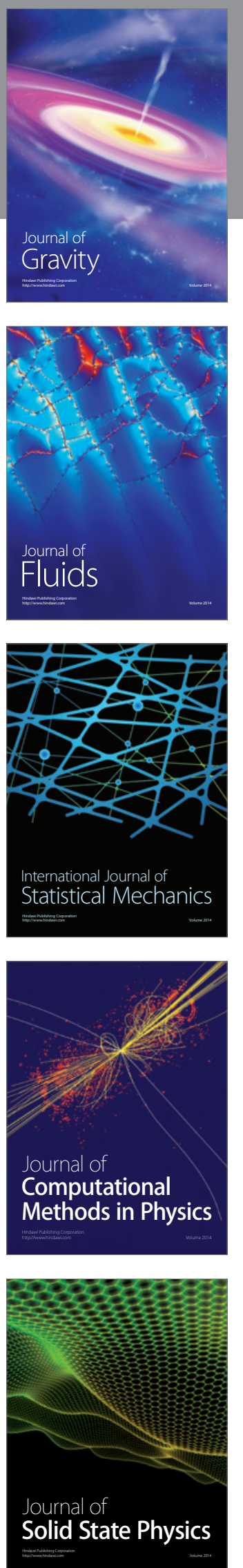





The Scientific World Journal
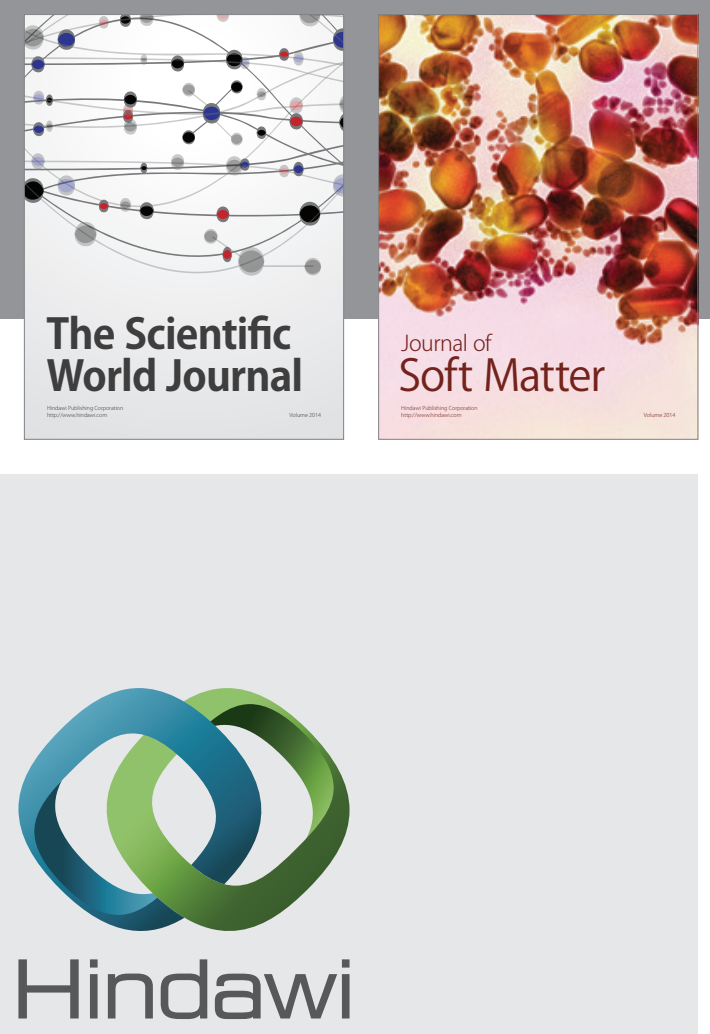

Submit your manuscripts at

http://www.hindawi.com

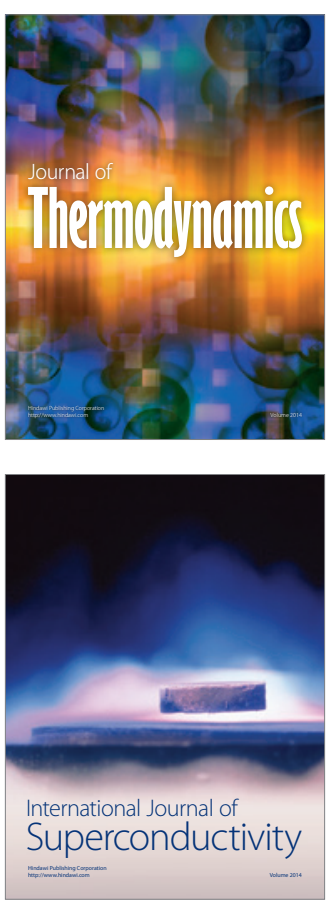
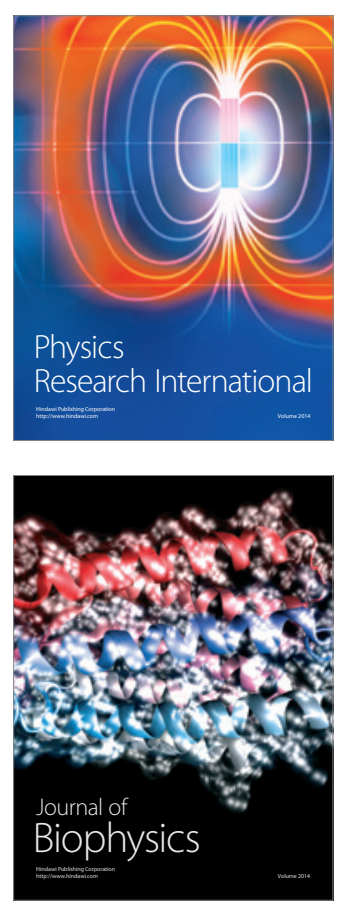
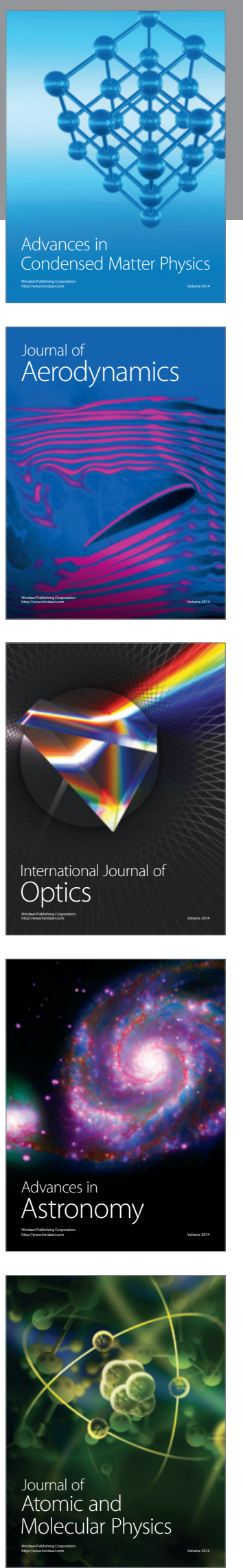